\title{
The Distribution of Pregnant Women with Different Pregnancy Risks - 4 Cities, China, 2019
}

\author{
Yanhui Liu'; Rong Luo'; Aiqun Huang ${ }^{1, *}$
}

\begin{abstract}
Summary
What is already known on this topic?

Based on different pregnancy risk levels, the implementation of the "Five-Color Management" for pregnant women can prevent adverse pregnancy outcomes and ensure the safety of mothers and infants. What is added by this report?

The proportions of being multipara and of advanced maternal age in the 4 cities (Beijing, Chengdu, Shenzhen, and Wuhan) were $47.4 \%$ and $13.3 \%$, respectively. The proportions of "Yellow and above" pregnancy risk ranged from $54.5 \%$ to $65.0 \%$ and ranged from $7.4 \%$ to $16.3 \%$ for "Orange and above" pregnancy risk. Among women with "Orange and above" pregnancy risk, most of them gave birth in public tertiary institutions $(71.8 \%-79.4 \%)$.

What are the implications for public health practice?

The implementation of the "Five-Color Management" for pregnant women with different pregnancy risks should be strengthened, especially those with "Orange and red" pregnancy risk who should be hospitalized for delivery in tertiary medical institutions if they have conditions.
\end{abstract}

In October 2015, China's One-Child Policy was replaced by a universal Two-Child Policy; following the policy change, multiparous births exceeded primiparous births nationwide (1). From July 2016 to December 2017, the monthly mean percentage of being multipara and being of advanced maternal age (aged 35 years and over) increased by $9.1 \%$ and $5.8 \%$, respectively, and this increase was also seen in at-risk pregnant women (2). The comprehensive high-risk score method for pregnant women was used to screen pregnancy risks, but the screening results were untargeted and disorganized (3). In order to prevent adverse pregnancy outcomes and ensure the safety of mothers and infants, the National Health Commission (NHC) of China in 2017 proposed implementing the "Five-Color Management" for women during pregnancy and 42-days postpartum based on different pregnancy risk levels (4). Compared with the traditional comprehensive high-risk score method, the "Five-Color Management" for pregnant women is to screen, assess, classify, and manage pregnancy risks from a thematic perspective (5). We conducted this study based on surveillance projects from 4 cities (Beijing, Chengdu, Shenzhen, and Wuhan) in China from 2014 to 2019. This study aims to analyze the implementation of the "Five-Color Management" in 2019 in the 4 cities. The results showed that the proportions of "Yellow and above" pregnancy risk ranged from $54.5 \%$ to $65.0 \%$ and ranged from $7.4 \%$ to $16.3 \%$ for "Orange and above" pregnancy risk. Among women with "Orange and above" pregnancy risk, most of them gave birth in public tertiary institutions (71.8\%-79.4\%).

The data were collected from the surveillance project that was founded by the China-World Health Organization Biennial Collaborative Projects entitled "Surveillance of high-risk maternal health services and management" (2018-2019). The surveillance project was implemented by the National Center for Women and Children's Health of China CDC, and its details were described in our previous publications (6-7). Briefly, three cities including Chengdu, Wuhan, and Shenzhen were selected for the surveillance from the western, central, and eastern regions of China, respectively. These cities were selected based on their geographical location, and the existing city-wide unified reporting system for maternal and child health $(\mathrm{MCH})$ covering the whole city. Furthermore, these criteria were used to select two districts (Haidian District and Chaoyang District) in Beijing to represent the northern region of China. The surveillance covered all medical institutions providing childbirth services in the 4 cities, including general hospitals, maternal and children's healthcare hospitals, specialized hospitals, community health centers/township hospitals, and private hospitals. In 2019, a total of 325 medical institutions were covered in the surveillance.

According to national regulations, the "Five-Color 
Management" requires that pregnant women be classified into five colors levels - green, yellow, orange, red, and purple - according to their basic conditions and pregnancy complications (4-5). "Green" indicates normal pregnancy; "Yellow" indicates general risk and that pregnancy complications are mild and stable; "Orange" indicates medium risk and that pregnancy complications pose a threat to mother and infant safety; "Red" indicates high risk and that pregnant women suffer from serious pregnancy complications, continued pregnancy may endanger the life of mother and infant; and "Purple" indicates that the pregnant women have infectious diseases such as viral hepatitis, syphilis, HIV/AIDS, tuberculosis, serious infectious pneumonia, or a specific viral infection (H1N7, Zika, etc.). Except for the combination of "Purple" and other color levels, pregnant women will be managed according to their "color level" with the highest pregnancy risks. "Yellow and above" was classified as high-risk pregnancy, and "Orange or above" was classified as key groups of highrisk pregnancy. In the 4 cities, the $\mathrm{MCH}$ information system covered the whole city, and individual antenatal care and delivery information for all pregnant women were recorded in the system. Due to the adjustment of the $\mathrm{MCH}$ information system, the analysis of the "Five-Color Management" only covered pregnant women from July 1 to December 31, 2019 in Wuhan, while in the other 3 cities included all pregnant women during 2019.

Among 625,305 pregnant women in the 4 cities, the proportions of being multipara and of advanced maternal age were $47.4 \%$ and $13.3 \%$, respectively. The proportion of being of advanced maternal age in multipara $(21.8 \%)$ was higher than that in primipara (5.7\%). (Table 1)

In the 4 cities, the proportion of "Yellow" pregnancy risk was between $48.1 \%$ and $62.1 \%$, and the proportion of "Orange" and "Red" pregnancy risk was the highest in Beijing (14.5\%) and the lowest in Wuhan $(6.3 \%)$. The proportion of "Yellow and above" pregnancy risk (high-risk pregnancy) ranged from $54.5 \%$ to $71.4 \%$, and "Orange and above" pregnancy risk (the key groups of high-risk pregnancy) ranged from $7.4 \%$ to $16.3 \%$. (Table 2 )

In the 4 cities, the proportion of pregnant women giving birth in public tertiary institutions was between $65.9 \%$ to $76.9 \%$, and the proportion giving birth in public secondary institutions or other institutions were between $11.2 \%$ to $18.6 \%$. More than $82.0 \%$ of pregnant women with "Yellow" risk gave birth in public secondary or above institutions, and $71.8 \%$ to $79.4 \%$ of pregnant women with "Orange and above" risk gave birth in public tertiary institutions. However,

TABLE 1. The number and proportions of multipara and advanced maternal age in 4 cities in China, 2019.

\begin{tabular}{lcrrrrr}
\hline \multirow{2}{*}{ Cities } & \multirow{2}{*}{ Pregnant women (N) } & Primipara (N/\%) & Multipara (N/\%) & \multicolumn{3}{c}{ Advanced maternal age } \\
\cline { 5 - 7 } & & & & Total (N/\%) & Primipara (N/\%) & Multipara (N/\%) \\
\hline Beijing & 84,403 & $54,774(64.9)$ & $29,629(35.1)$ & $17,317(20.5)$ & $6,306(11.5)$ & $11,011(37.2)$ \\
Chengdu & 214,554 & $115,744(53.9)$ & $98,810(46.1)$ & $19,998(9.3)$ & $4,285(3.7)$ & $15,713(15.9)$ \\
Shenzhen & 208,167 & $90,713(43.6)$ & $117,454(56.4)$ & $31,744(15.2)$ & $5,060(5.6)$ & $26,684(22.7)$ \\
Wuhan & 118,181 & $67,408(57.0)$ & $50,773(43.0)$ & $14,284(12.1)$ & $2,970(4.4)$ & $11,314(22.3)$ \\
Total & 625,305 & $328,639(52.6)$ & $296,666(47.4)$ & $83,343(13.3)$ & $18,621(5.7)$ & $64,722(21.8)$ \\
\hline
\end{tabular}

TABLE 2. The distribution of different pregnancy risks according to color levels in 4 cities in China, 2019.

\begin{tabular}{|c|c|c|c|c|c|c|c|}
\hline \multirow{2}{*}{ Cities } & \multicolumn{5}{|c|}{ Different pregnancy risks* (N/\%) } & \multirow{2}{*}{$\begin{array}{c}\text { Yellow and above } \\
\text { (N/\%) }\end{array}$} & \multirow{2}{*}{$\begin{array}{l}\text { Orange and abov } \\
(\mathrm{N} / \%)\end{array}$} \\
\hline & Green & Yellow & Orange & Red & Purple & & \\
\hline Beijing & $28,935(34.3)$ & $42,801(50.7)$ & $11,165(13.2)$ & $1,078(1.3)$ & $1,761(2.1)$ & $55,468(65.7)$ & $13,729(16.3)$ \\
\hline Chengdu & $74,972(34.9)$ & $123,301(57.6)$ & $15,171(7.0)$ & $384(0.2)$ & $2,372(1.1)$ & $139,582(65.1)$ & $17,671(8.2)$ \\
\hline Shenzhen & $59,574(28.6)$ & $129,170(62.1)$ & $17,923(8.6)$ & $1,142(0.5)$ & $1,597(0.8)$ & $148,593(71.4)$ & $20,449(9.8)$ \\
\hline Wuhan $^{\dagger}$ & $28,068(45.5)$ & $29,679(48.1)$ & $3,548(5.8)$ & $300(0.5)$ & $811(1.3)$ & $33,616(54.5)$ & $4,537(7.4)$ \\
\hline
\end{tabular}

* "Green" indicates normal pregnancy. "Yellow" indicates general risk and that pregnancy complications are mild and stable. "Orange" indicates medium risk and that pregnancy complications pose a threat to mother and infant's safety. "Red" indicates high risk and that pregnant women suffer from serious pregnancy complications, continued pregnancy may endanger the life of mother and infant. "Purple" indicates that the pregnant women had an infectious disease such as viral hepatitis, syphilis, HIV infection and AIDS, tuberculosis, serious infectious pneumonia, or a specific viral infection (H1N7, Zika, etc.).

†The analysis of the "Five-Color Management" in Wuhan only included pregnant women from July 1 to December 31, 2019. 
more than $20 \%$ of pregnant women that had "Orange and above" risk gave birth in public secondary and below institutions. (Table 3)

\section{DISCUSSION}

The surveillance project carried out in 4 relatively developed cities of China in 2019 showed that the proportions of pregnant women with "Yellow and above" risk and "Orange and above" risk were higher and that most of them received the "Five-Color Management" based on different pregnancy risks. However, some pregnant women with higher risk still gave birth in lower-level institutions. Therefore, the implementation of the "Five-Color Management" for pregnant women with different risks should be further strengthened, especially for those with "Orange" and "Red" risk who should be hospitalized for delivery in tertiary medical institutions if they have conditions.

The literature showed that multipara and advanced maternal age were associated with increased pregnancy complications, in turn leading to maternal and fetal morbidity and mortality (8-9). In this study, the proportions of being multipara and of advanced maternal age in the 4 cities were $47.4 \%$ and $13.3 \%$, respectively, which were basically consistent with the national levels in $2019(49.9 \%$ and $15.8 \%$, respectively) (10). Therefore, more attention should be paid to the "Five-Color Management" for pregnant women to improve the health of pregnant women that were multipara or of advanced maternal age and reduce maternal and perinatal mortality.

The NHC requires secondary and above medical institutions to assess and classify pregnancy risks for all pregnant women, and tertiary medical institutions to assess pregnancy suitability for "Red" pregnancy risk. "Yellow" pregnancy risk women are recommended to hospitalization for delivery in secondary or above institutions; "Orange and above" pregnancy risk should be hospitalized for delivery in tertiary institutions if they have conditions; "Purple" pregnancy risk should be managed according to the regulations on the prevention and treatment of infectious diseases (4-5). The pilot results showed that the "Five-Color Management" for pregnant women was effective in women accessing emergency obstetric care, especially for receipt of appropriate care once a medical institution is reached $(11-12)$.

Our findings suggested the proportions of pregnant women with "Yellow and above" and "Orange and above" risk in our sample were higher than that in Guangdong Province in 2018 (42.2\% and 6.9\%, respectively) (12). Most pregnant women with different risk levels were hospitalized for delivery in appropriate level institutions, but there were still some pregnant women with higher risk levels that gave birth in lower-level institutions. In order to stabilize and reduce the pregnancy risk, antenatal care, follow-up management, and timely treatment of pregnancy

TABLE 3. The distribution of delivery with different pregnancy risks in 4 cities in China, 2019.

\begin{tabular}{|c|c|c|c|c|c|}
\hline \multirow{2}{*}{ Cities } & \multirow{2}{*}{ Institutions ${ }^{*}$} & \multicolumn{3}{|c|}{ Different pregnancy risks (N/\%) } & \multirow{2}{*}{ Total (N/\%) } \\
\hline & & Green & Yellow & Orange and above & \\
\hline \multirow[t]{3}{*}{ Beijing } & Public tertiary & $21,232(73.4)$ & $24,520(58.7)$ & $9,862(71.8)$ & $55,614(65.9)$ \\
\hline & Public secondary & $3,554(12.3)$ & $10,048(24.1)$ & $2,114(15.4)$ & $15,716(18.6)$ \\
\hline & Others & $4,149(14.3)$ & $7,171(17.2)$ & $1,753(12.8)$ & $13,073(15.5)$ \\
\hline \multirow[t]{3}{*}{ Chengdu } & Public tertiary & $55,969(74.7)$ & $85,152(69.8)$ & $13,937(78.9)$ & $155,058(72.3)$ \\
\hline & Public secondary & $9,314(12.4)$ & $16,939(13.9)$ & $1,639(9.3)$ & $27,892(13.0)$ \\
\hline & Others & $9,689(12.9)$ & $19,820(16.3)$ & 2,095 (11.9) & $31,604(14.7)$ \\
\hline \multirow[t]{3}{*}{ Shenzhen } & Public tertiary & $43,226(72.1)$ & $92,332(72.1)$ & $15,936(79.4)$ & $151,494(72.8)$ \\
\hline & Public secondary & $8,512(14.2)$ & $16,702(13.0)$ & $2,048(10.2)$ & $27,262(13.1)$ \\
\hline & Others & $8,206(13.7)$ & $19,110(14.9)$ & $2,095(10.4)$ & $29,411(14.1)$ \\
\hline \multirow[t]{3}{*}{ Wuhan $^{\dagger}$} & Public tertiary & $21,297(75.9)$ & $22,707(78.1)$ & $3,415(75.3)$ & $47,419(76.9)$ \\
\hline & Public secondary & $3,783(13.5)$ & $3,128(10.8)$ & $435(9.6)$ & $7,346(11.9)$ \\
\hline & Others & $2,988(10.6)$ & $3,244(11.2)$ & 687 (15.1) & $6,919(11.2)$ \\
\hline
\end{tabular}

${ }^{*}$ Medical institutions providing childbirth services were divided into public tertiary, public secondary, and other institutions. Other institutions were all health facilities providing childbirth services except public tertiary and public secondary including public primary hospitals, private midwifery hospitals, etc.

†The analysis of the "Five-Color Management" in Wuhan only included pregnant women from July 1 to December 31, 2019. 
complications should be strengthened. Therefore, medical institutions providing childbirth services should strengthen the implementation of the "FiveColor Management" for pregnant women, especially for pregnant women with "Orange" and "Red" risk levels who should be transferred to tertiary institutions for antenatal care and delivery.

This study were subject to certain limitations. First, the surveillance data were collected in 4 cities with an existing city-wide unified reporting system for $\mathrm{MCH}$, so the results might not fully be representative of the regional and national levels. Second, there is no comparison between the results of the "Five-Color" classification and pregnancy outcomes. In the future, more studies should be taken to fix this issue that may have further value for antenatal care and hospital delivery.

doi: $10.46234 / \mathrm{ccdcw} 2021.016$

\# Corresponding author: Aiqun Huang, aqhuang@chinawch.org.cn.

${ }^{1}$ National Center for Women and Children's Health, Chinese Center for Disease Control and Prevention. Beijing, China.

Submitted: September 12, 2020; Accepted: January 11, 2021

\section{REFERENCES}

1. Li PC, Liao ZJ, Wang C, Zhou YB, Li HT, Chen DJ, et al. Maternal characteristics and birth outcomes under different family planning policy. Chin J Reprod Health 2017;28(6):501 - 5. http://d.wan fangdata.com.cn/periodical/zgsyjkzz201706001. (In Chinese).

2. Li HT, Xue M, Hellerstein S, Cai Y, Gao YQ, Zhang YL, et al. Association of China's universal two child policy with changes in births and birth related health factors: national, descriptive comparative study. BMJ 2019;366:14680. http://dx.doi.org/10.1136/bmj.14680.
3. Zhang SS, Huang T. Value of improved risk early warning and evaluation management on improving perinatal outcome of high-risk pregnant women. Chin Med Rec 2019;20(2):91 - 4. https://kns.cnki. net/KCMS/detail/detail.aspx?FileName=ZGBN201902032\&DbName =CJFQ2019. (In Chinese).

4. National Health Commission of the People's Republic of China. Regulations on pregnancy risk assessment and management (2017 No.35). http://www.nhc.gov.cn/fys/s3581/201711/9c3dc9b4a8494d9a 94c02f890e5085b1.shtml. [2020-12-8]. (In Chinese).

5. National Health Commission of the People's Republic of China. Notice on strengthening the work of safeguarding maternal and infant safety (2017 No.42). https://www.chinafpa.org.cn/zcfg/xgfg/201901/t2019 0125_42951.html. [2020-12-8]. (In Chinese).

6. Yan S, Huang AQ, Xu T, Hu HQ, Zhao W, Ma WX, et al. Analysis of obstetricians' workload of institutions of maternal and children's healthcare in some Chinese large and medium-sized cities. Chin J Reprod Health 2008;29(4):319 - 23. https://kns.cnki.net/KCMS/detail/ detail.aspx?FileName=SYJK201804004\&DbName=CJFQ2018. (In Chinese).

7. Huang AQ, Liu YH, Jin X. The impact of new adjustments to the family planning policy on the number of live births in four developed urban areas - China, 2013-2019. China CDC Wkly 2020;2(28): 530 - 3. http://weekly.chinacdc.cn/en/article/doi/10.46234/ccdcw2020. 143.

8. Care AS, Bourque SL, Morton JS, Hjartarson EP, Davidge ST. Effect of advanced maternal age on pregnancy outcomes and vascular function in the rat. Hypertension 2015;65(6):1324 - 30. http://dx.doi.org/10.1161/ HYPERTENSIONAHA.115.05167.

9. Zhao YY, Yuan PB, Chen L. Problems faced by advanced maternal age in the age of two children. Chin J Pract Gynecol Obstet 2020;36(2): 97 - 100. https://kns.cnki.net/KCMS/detail/detail.aspx?FileName=ZG SF202002001\&DbName=CJFQ2020. (In Chinese).

10. Department of Maternal and Child Health of the National Health Commission. Analysis report on the national maternal and child health information for 2020. [2020-12-8]. (In Chinese).

11. Knight HE, Self A, Kennedy SH. Why are women dying when they reach hospital on time? a systematic review of the 'third delay'. PLoS One 2013;8(5):e63846. http://dx.doi.org/10.1371/journal.pone.006 3846.

12. Wu YT, Chen Q, Liao HZ, Wu L, Wang ZQ, Xu HL, et al. Matching degree between the classification of maternal pregnancy risk and the level of midwifery institutions, Guangdong. Mod Prev Med 2020;47(1):84 - 7. http://d.wanfangdata.com.cn/periodical/xdyfyx20 2001020. (In Chinese). 